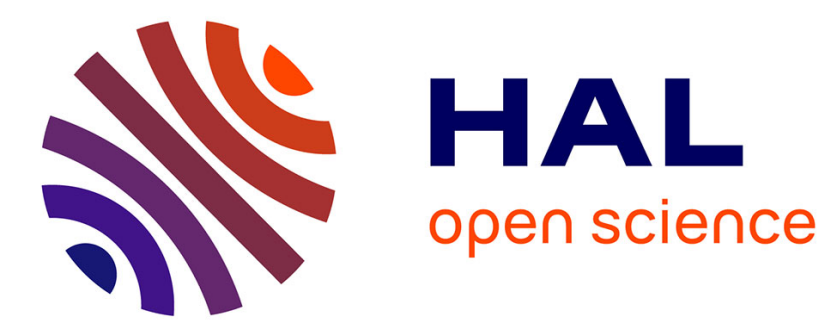

\title{
Experimental violation of Tsirelson's bound by Maxwell fields
}

\author{
N. Sandeau, Hassan Akhouayri, A. Matzkin, Thomas Durt
}

\section{To cite this version:}

N. Sandeau, Hassan Akhouayri, A. Matzkin, Thomas Durt. Experimental violation of Tsirelson's bound by Maxwell fields. Physical Review A : Atomic, molecular, and optical physics [1990-2015], 2016, 93 (5), pp.053829. 10.1103/PhysRevA.93.053829 . hal-01321140

\section{HAL Id: hal-01321140 \\ https://hal.science/hal-01321140}

Submitted on 26 May 2016

HAL is a multi-disciplinary open access archive for the deposit and dissemination of scientific research documents, whether they are published or not. The documents may come from teaching and research institutions in France or abroad, or from public or private research centers.
L'archive ouverte pluridisciplinaire HAL, est destinée au dépôt et à la diffusion de documents scientifiques de niveau recherche, publiés ou non, émanant des établissements d'enseignement et de recherche français ou étrangers, des laboratoires publics ou privés. 


\title{
Experimental violation of Tsirelson's bound by Maxwell fields
}

\author{
N. Sandeau, ${ }^{1}$ H. Akhouayri, ${ }^{1}$ A. Matzkin,${ }^{2}$ and T. Durt ${ }^{1, *}$ \\ ${ }^{1}$ Aix Marseille Université, CNRS, Centrale Marseille, Institut Fresnel UMR 7249, 13013 Marseille, France \\ ${ }^{2}$ Laboratoire de Physique Théorique et Modélisation (CNRS Unité 8089), Université de Cergy-Pontoise, 95302 Cergy-Pontoise cedex, France
}

(Received 27 November 2015; published 23 May 2016)

\begin{abstract}
In analogy with quantum optics it is possible to impose nonseparability between different degrees of freedom of an optical beam. The resulting correlations between these degrees of freedom can be investigated with correlations functions traditionally employed in quantum mechanics, such as the well-known Clauser-Horne-Shimony-Holt (CHSH) correlation function. In this paper we present results achieving a maximal violation of Tsirelson's bound on CHSH correlations between spatial and polarization degrees of freedom of classical (Maxwell) fields. We describe the theoretical method, based on the realization of a nonunitary gate, and then proceed to its experimental implementation carried out with classical optical techniques. Our approach relies on the realization at the level of classical Maxwell fields of a so-called POVM (positive operator valued measure) which is traditionally discussed in the realm of quantum physics.
\end{abstract}

DOI: 10.1103/PhysRevA.93.053829

\section{INTRODUCTION}

Entanglement is often said to be, following Schrödinger's celebrated words, the characteristic trait of quantum mechanics [1]. Nevertheless, it was realized, less than two decades ago [2,3], that classical Maxwell fields could also present entanglement. Of course, the physical origin and meaning of entanglement in this context is quite different from what entanglement encompasses in quantum mechanics: we are not dealing here with quantum states of several particles, but with degrees of freedom of classical electromagnetic fields, such as polarization or spatial parity.

When the full state of the electromagnetic field cannot be written as a tensor product of these degrees of freedom, the corresponding beam does not have a well defined state for the relevant properties (e.g., it is not polarized, nor is it endowed with a given spatial parity). The well-known tools employed for quantum correlations can then be exported to investigate correlations between coupled, nonseparable degrees of freedom in Maxwell fields. Formally, this is grounded on the common structural features afforded by the Hilbert spaces employed in both cases [4]. Recently several groups started performing experiments in which the various measures of quantum entanglement have appeared as a useful tool for evaluating quantitatively correlations between degrees of freedom of classical light fields. Most of these works have dealt with the violation of Bell inequalities [5-8], or with related measures of coherence properties by optical beams [9-13].

In a quantum context, the violation of the Bell inequalities obtained with entangled states indicates that the quantum correlations are stronger than those allowed by local variables. However, as is well known, quantum correlations are also bounded, by what is known as Tsirelson's bound $[14,15]$. In principle, one could envisage stronger than quantum correlations: they would then violate Belltype inequalities by a value larger than Tsirelson's bound. A model giving rise to "superquantum" correlations was

\footnotetext{
*thomas.durt@centrale-marseille.fr
}

proposed some time ago by Popescu and Rohrlich [16]. Actually the so-called PR model achieves the maximal possible value for correlations, still respecting the no-signalling condition.

In this work, we investigate the possibility of violating Bell-type inequalities with Maxwell beams beyond Tsirelson's bound. We report below experiments involving electromagnetic fields nonseparable in the polarization and spatial parity degrees of freedom that achieve PR correlations. This is done by implementing a Maxwell fields version of a probabilistic nonunitary gate [17]. From this point of view, the classical fields setup proposed below would correspond, in a quantum description, to a POVM aimed at probabilistically achieving a nonunitary transformation.

The paper is structured as follows. In Sec. II, we briefly recall the properties of classical, quantum, and superquantum (or PR) correlations, and we put them in relation with Tsirelson bound. We show in Sec. III how entangled Maxwell beams make it possible to saturate the Tsirelson bound, in line with previous works [5-13]. In Sec. IV, we show how to implement maximal PR correlations by following a method [17] proposed originally in a quantum context in order to realize a probabilistic nonunitary gate. We will see that a simple classical optical setup provides an optimal realization of the method. In Sec. V, we describe the experimental setup and present the experimental results. Section VI is devoted to discussing the results, in particular by drawing a comparison with previous quantum optical realizations of nonunitary gates and suggesting a link between the quantum regime (discrete photon counters, few photons at a time) and our results obtained in the classical regime (many photons at a time, measured with continuous intensity detectors). A conclusion is provided at the end (Sec. VII).

\section{PR CORRELATIONS AND TSIRELSON BOUND}

Let us consider two dichotomic quantities (for instance spin or polarization) $A$ and $B$ with possible outcomes: $A=+1$ or $A=-1, B=+1$ or $B=-1$. Let us denote by $P_{A B}(\alpha, \beta)$ the four probabilities for the outcomes, where $\alpha$ and $\beta$ label settings relative to the measurements of $A$ and $B$ respectively. 
Then an average can be defined through

$$
\mathcal{E}(\alpha, \beta)=\sum_{A= \pm 1} \sum_{B= \pm 1} A B P_{A B}(\alpha, \beta) .
$$

We can go further and introduce a function indicating how the measured values of $A$ and $B$ are correlated. The ClauserHorne-Shimony-Holt (CHSH) [18] function $\mathcal{C}\left(\alpha, \alpha^{\prime} ; \beta, \beta^{\prime}\right)$ provides a useful measure of the correlations between the outcomes of $A$ and $B$, in a situation where two different dichotomic measures are realized on each of them ( $\alpha$ and $\alpha^{\prime}$ on $A, \beta$ and $\beta^{\prime}$ on $B$ ),

$$
\begin{aligned}
\mathcal{C}\left(\alpha, \alpha^{\prime} ; \beta, \beta^{\prime}\right) \equiv & \left|\mathcal{E}(\alpha, \beta)-\mathcal{E}\left(\alpha, \beta^{\prime}\right)\right|+\mid \mathcal{E}\left(\alpha^{\prime}, \beta\right) \\
& +\mathcal{E}\left(\alpha^{\prime}, \beta^{\prime}\right) \mid .
\end{aligned}
$$

Four important results are known about the CHSH function:

(1) If the probabilities $P_{A B}(\alpha, \beta)$ are seen as averages obtained from an unspecified underlying elementary mechanism ruled by a hidden variable $\lambda$ endowed with a statistical distribution $\mu(\lambda)$, that is $P_{A B}(\alpha, \beta)=\int p_{A B}(\alpha, \beta, \lambda) \mu(\lambda) d \lambda$, then when the probabilities of these elementary processes are independent, i.e., $p_{A B}(\alpha, \beta, \lambda)=p_{A}(\alpha, \lambda) p_{B}(\beta, \lambda)$, the $\mathrm{CHSH}$ function is bounded by $2, \mathcal{C}\left(\alpha, \alpha^{\prime} ; \beta, \beta^{\prime}\right) \leqslant 2$. This bound is often called the "classical" bound, following Bell's discussion of these probabilities in a context involving constraints imposed by local causality on spacelike measurements [19].

(2) In quantum mechanics, when $A$ and $B$ are measured on separable systems (roughly, when the results of the measurements carried out on $A$ and $B$ are independent of each other), the maximal value of the CHSH function is 2 . Actually, in this case the probability $P_{\mathrm{xy}}$ is a weighted sum of factorizable probabilities ( $P_{\mathrm{xy}}^{\mathrm{fact}}=P_{x} P_{y}$, with $x=A$ or $A^{\prime}$, $y=B$ or $B^{\prime}$ ), and it is easy to check that, by construction, the $\mathrm{CHSH}$ function is upperly bounded by 2 , by simple algebric manipulations. A quantum system is said to be separable if and only if it is a weighted sum (mixture) of factorizable pure states. Quantum separable systems never violate the classical bound $\mathcal{C}\left(\alpha, \alpha^{\prime} ; \beta, \beta^{\prime}\right) \leqslant 2$.

(3) Quantum entangled systems may violate the classical bound, provided they are prepared in well-chosen entangled states, and provided the binary observables corresponding to the measures (of $\alpha$ and $\alpha^{\prime}$ on $A, \beta$ and $\beta^{\prime}$ on $B$ ) are chosen accordingly [20]. However, the maximal violation reachable with quantum systems obeying unitary evolution laws is $\mathcal{C}\left(\alpha, \alpha^{\prime} ; \beta, \beta^{\prime}\right) \leqslant 2 \sqrt{2}$. This constitutes the so-called Tsirelson bound [14].

(4) The no-signaling condition prohibits the possibility to make use of quantum correlations in order to transfer classical information (faster than light in situations where we consider nonlocal correlations). It can be expressed as follows: for any $x, y, X, Y, Y^{\prime}$,

$$
\begin{aligned}
P(x)_{X, Y} & \equiv P(x, y=+1)_{X, Y}+P(x, y=-1)_{X, Y} \\
& =P(x)_{X, Y^{\prime}}, \\
P(y)_{X, Y} & \equiv P(x=+1, y)_{X, Y}+P(x=-1, y)_{X, Y} \\
& =P(y)_{X^{\prime}, Y},
\end{aligned}
$$

where $X\left(X^{\prime}\right)$ is any dichotomic measure on the $A$ system, and $x\left(x^{\prime}\right)$ its result $( \pm 1)$, while $Y\left(Y^{\prime}\right)$ is any dichotomic measure on the $B$ system, and $y\left(y^{\prime}\right)$ its result $( \pm 1)$. In other words, the "local" probabilities assigned to measures carried out on one of the subsystems only do not depend on which measure is carried out on the second system. Quantum correlations respect the no-signaling condition, and they also obey Tsirelson's bound as discussed above. However, the maximal possible value of the $\mathrm{CHSH}$ (2) function is 4 [because the value of $\mathcal{E}(\alpha, \beta)$ given by Eq. (1) is comprised between -1 and +1$]$. As has been shown by Popescu and Rohrlich [16], it is possible to find "superquantum" correlations (that we shall denote PR correlations) that respect the no-signaling condition and maximally violate Tsirelson's bound. We denote maximal PR correlations those which reach the ultimate bound $\mathcal{C}\left(\alpha, \alpha^{\prime} ; \beta, \beta^{\prime}\right)=4$ (by example, what is called PR box in the literature [16] is a hypothetical device that delivers maximal PR correlations).

As we shall show below, Maxwell fields may simulate the PR box. We first review and discuss the CHSH correlation function for classical electromagnetic fields.

\section{SATURATION OF TSIRELSON BOUND BY MAXWELL FIELDS}

We introduce in this section a CHSH inequality that can be obtained with a classical optical beam coupling two degrees of freedom. We will employ essentially the same degrees of freedom of a classical optical beam as the ones employed in Ref. [10], namely the polarization and the spatial parity along one of the transverse directions (say $x$ ). The parity, when defined, can therefore be positive $[E(x)=E(-x)$ and $\pi=+1 \equiv \pi+]$ or negative $[E(x)=-E(-x)$ and $\pi=-1 \equiv$ $\pi-]$. In this section we discuss operations that allow the correlations to reach Tsirelson's bound; operations leading to violations of this bound will be seen in Secs. IV-VI.

\section{A. Preparation}

The initial beam is taken to be linearly polarized along the diagonal direction in a horizontal-vertical polarization basis, with a spatial part of positive parity. If we introduce, in a bra-ket quantumlike notation, the basis $(|H, \pi+\rangle,|H, \pi-\rangle,|V, \pi+\rangle,|V, \pi-\rangle)$, this initial state can be written in the form

$$
(1 / \sqrt{2})(|V, \pi+\rangle+|H, \pi+\rangle) .
$$

In classical optics, this state would be characterized by a Jones vector (describing the polarization as a vector in the $V$ and $H$ basis) given by

$$
E_{0}=\frac{1}{\sqrt{2}}\left(\begin{array}{l}
1 \\
1
\end{array}\right)
$$

with the spatial part of positive parity, i.e.,

$$
E_{0}=\frac{1}{\sqrt{2}}\left(\begin{array}{l}
f_{\pi=1}(x) \\
f_{\pi=1}(x)
\end{array}\right) .
$$

When writing Jones vectors, we will not write explicitly the spatial part and assume we are dealing with a function of spatial positive parity: hence Eq. (6) will actually denote (7). Note that a function of negative parity is obtained through $f_{\pi=-1}(x)=\operatorname{sgn}(x) f_{\pi=1}(x)$. We now apply to (5) the unitary 
transformation

$$
|V, \pi+\rangle\langle V, \pi+|-| H, \pi-\rangle\langle H, \pi+|
$$

that is the $H$ polarization axis gets a spatial parity flip from positive to negative, while the field component on the $V$ axis is left untouched. In terms of Jones matrices this means that we apply to (6) the transformation represented by the generalized [21] Jones matrix

$$
\left(\begin{array}{cc}
1 & 0 \\
0 & -\operatorname{sgn}(x)
\end{array}\right)
$$

This gives a coupled beam of the form

$$
(1 / \sqrt{2})(|V, \pi+\rangle-|H, \pi-\rangle),
$$

which reads, in the Jones vector notation,

$$
E_{i}=\frac{1}{\sqrt{2}}\left(\begin{array}{c}
1 \\
-\operatorname{sgn}(x)
\end{array}\right)
$$

for which neither the parity nor the polarization can be defined independently (the parity is negative on the $H$ component but positive on the $V$ component). The state described in (10) and (11) is analog to the, say, two particles spin-1/2 singlet state of quantum mechanics. Instead of dealing with an entangled state of two spins, we have here two coupled degrees of freedom of the beam.

\section{B. Analysis}

The beam analysis is the counterpart of the spin measurements on the singlet state. Instead of measuring spin projections for chosen angles $\alpha$ and $\beta$ for particles 1 and 2 respectively, the analysis involves measuring relative intensities in the following way.

(i) For the polarization: the beam is rotated by an angle $-\alpha / 2$ and then one measures the components of the field $E_{V}(\alpha)$ and $E_{H}(\alpha)$ along the $V$ and $H$ axes respectively, regardless of the parity.

(ii) For the spatial parity: the beam is rotated by an angle $\beta / 2$ and then the components $E_{\pi+}(\beta)$ and $E_{\pi-}(\beta)$ are obtained by filtering the beam according to the parity.

After both transformations the prepared signal $E_{i}$ becomes

$$
E_{f}=\frac{1}{\sqrt{2}}\left(\begin{array}{c}
\cos \left(\frac{\alpha-\beta}{2}\right)-\operatorname{sgn}(x) \sin \left(\frac{\alpha-\beta}{2}\right) \\
-\sin \left(\frac{\alpha-\beta}{2}\right)-\operatorname{sgn}(x) \cos \left(\frac{\alpha-\beta}{2}\right)
\end{array}\right) .
$$

Employing the aforementioned bra-ket quantumlike notation, in a basis $(|H, \pi+\rangle,|H, \pi-\rangle,|V, \pi+\rangle,|V, \pi-\rangle)$ the rotation and phase-shift operations are described by the matrices

$$
\mathcal{R}(\alpha)=\left(\begin{array}{cccc}
\cos \frac{\alpha}{2} & 0 & -\sin \frac{\alpha}{2} & 0 \\
0 & \cos \frac{\alpha}{2} & 0 & -\sin \frac{\alpha}{2} \\
\sin \frac{\alpha}{2} & 0 & \cos \frac{\alpha}{2} & 0 \\
0 & \sin \frac{\alpha}{2} & 0 & \cos \frac{\alpha}{2}
\end{array}\right)
$$

and

$$
\mathcal{P}(\beta)=\left(\begin{array}{cccc}
\cos \frac{\beta}{2} & -\sin \frac{\beta}{2} & 0 & 0 \\
\sin \frac{\beta}{2} & \cos \frac{\beta}{2} & 0 & 0 \\
0 & 0 & \cos \frac{\beta}{2} & -\sin \frac{\beta}{2} \\
0 & 0 & \sin \frac{\beta}{2} & \cos \frac{\beta}{2}
\end{array}\right)
$$

respectively, acting on the initial state (11) now written

$$
v_{i}=\left(\begin{array}{c}
0 \\
-\frac{1}{\sqrt{2}} \\
\frac{1}{\sqrt{2}} \\
0
\end{array}\right) \text {. }
$$

After performing these operations, the following relative intensities relations hold:

$$
\begin{aligned}
& I_{H \pi+}(\alpha, \beta)=\frac{1}{2} \sin ^{2}\left(\frac{\alpha-\beta}{2}\right), \\
& I_{H \pi-}(\alpha, \beta)=\frac{1}{2} \cos ^{2}\left(\frac{\alpha-\beta}{2}\right), \\
& I_{V \pi+}(\alpha, \beta)=\frac{1}{2} \cos ^{2}\left(\frac{\alpha-\beta}{2}\right), \\
& I_{V \pi-}(\alpha, \beta)=\frac{1}{2} \sin ^{2}\left(\frac{\alpha-\beta}{2}\right),
\end{aligned}
$$

where $I_{H \pi+}(\alpha, \beta)$ is the intensity corresponding to the positive parity part of the $H$ component of the beam after having rotated the polarization by $\alpha / 2$, separated the $H / V$ components, and flipped the parity with a $\beta$-dependent phase shift (a similar analysis applies to the other three possibilities $\left.I_{H \pi-}, I_{V \pi+}, I_{V \pi-}\right)$.

Actually, the relative field intensities (16)-(19) are exactly equal to the probabilities obtained with a singlet quantum state. As those probabilities respect the no-signalling condition, we expect that a classical analog of the no-signalling condition is fulfilled here, which is well so, as can be seen from Eqs. (20)-(23),

$$
\begin{aligned}
& I_{H}(\alpha)=\frac{1}{2}\left[\sin ^{2}\left(\frac{\alpha-\beta}{2}\right)+\cos ^{2}\left(\frac{\alpha-\beta}{2}\right)\right]=\frac{1}{2}, \\
& I_{V}(\alpha)=\frac{1}{2}\left[\cos ^{2}\left(\frac{\alpha-\beta}{2}\right)+\sin ^{2}\left(\frac{\alpha-\beta}{2}\right)\right]=\frac{1}{2}, \\
& I_{\pi+}(\beta)=\frac{1}{2}\left[\cos ^{2}\left(\frac{\alpha-\beta}{2}\right)+\sin ^{2}\left(\frac{\alpha-\beta}{2}\right)\right]=\frac{1}{2}, \\
& I_{\pi-}(\beta)=\frac{1}{2}\left[\cos ^{2}\left(\frac{\alpha-\beta}{2}\right)+\sin ^{2}\left(\frac{\alpha-\beta}{2}\right)\right]=\frac{1}{2} .
\end{aligned}
$$

Formally we can pursue the analogy with quantum probabilities and define an average encapsulating the relative strengths of the intensities (16)-(19) by assigning a dichotomic value associated with the polarization components and another dichotomic value associated with the parity component. Let us thus associate $A=+1$ to the $H$ polarization component and $A=-1$ to the $V$ polarization component, and $B=+1$ to $\pi+$ and $B=-1$ to $\pi-$. Then an average can be defined through

$$
\begin{aligned}
\mathcal{E}(\alpha, \beta) & =\sum_{A= \pm 1} \sum_{B= \pm 1} A B I_{A B} \\
& =-\cos (\alpha-\beta) .
\end{aligned}
$$

We can pursue the analogy further and introduce a correlation function indicating how the polarization and the parity are correlated. The $\mathrm{CHSH}$ function

$$
\mathcal{C}\left(\alpha, \alpha^{\prime} ; \beta, \beta^{\prime}\right) \equiv\left|\mathcal{E}(\alpha, \beta)-\mathcal{E}\left(\alpha, \beta^{\prime}\right)\right|+\left|\mathcal{E}\left(\alpha^{\prime}, \beta\right)+\mathcal{E}\left(\alpha^{\prime}, \beta^{\prime}\right)\right|
$$


quantifies the correlations between the polarization and the spatial parity. The saturation of Tsirelson's bound corresponds in the present case to the values $\left(\alpha, \alpha^{\prime} ; \beta, \beta^{\prime}\right)=$ $(0, \pi / 2 ; \pi / 4,3 \pi / 4)$ for which we get $\mathcal{C}\left(\alpha, \alpha^{\prime} ; \beta, \beta^{\prime}\right)=$ $2 \sqrt{2}$.

\section{REALIZATION OF MAXIMAL PR CORRELATIONS}

\section{A. Extremal nonunitary gate}

One can imagine situations in which the "nonsignaling condition" is satisfied so that the measurement statistics of one property does not depend on the setting of the other measured property, but for which

$$
\mathcal{C}\left(\alpha, \tilde{\alpha}^{\prime} ; \beta, \tilde{\beta}^{\prime}\right)>2 \sqrt{2},
$$

where we introduced tilded values for referring to certain operations which cannot be described by Eqs. (13) and (14). It can be easily checked that the probability table, Table I (where as above $\alpha$ and $\beta$ are the angles referring to polarization and parity operations respectively), maximizes the $\mathrm{CHSH}$ correlation function.

It is worth noting here that these operations cannot be described by Eqs. (13) and (14). $\alpha=0, \beta=0$ still refer to the identity operator but $\tilde{\alpha}_{f}$ and $\tilde{\beta}_{f}$ refer to up to now unspecified operations, that will be operationally defined later. The pattern given in the probability table is identical to the one of the model introduced by Popescu and Rohrlich [16] to describe a "nonlocal"'[22] yet nonsignalling box (the so-called PR box). It is nonsignalling since

$$
\begin{gathered}
I_{H}\left(\alpha=0\left(\alpha=\tilde{\alpha}_{f}\right)\right)=\frac{1}{2}, \\
I_{V}\left(\alpha=0\left(\alpha=\tilde{\alpha}_{f}\right)\right)=\frac{1}{2}, \\
I_{\pi+}\left(\beta=0\left(\beta=\tilde{\beta}_{f}\right)\right)=\frac{1}{2}, \\
I_{\pi-}\left(\beta=0\left(\beta=\tilde{\beta}_{f}\right)\right)=\frac{1}{2} .
\end{gathered}
$$

This "box" is more nonlocal than what is allowed by quantum mechanics: with $\mathcal{E}(\alpha, \beta)$ defined from Eq. (24) we have $\mathcal{E}(0,0)=-1, \mathcal{E}\left(0, \tilde{\beta}_{f}\right)=1, \mathcal{E}\left(\tilde{\alpha}_{f}, 0\right)=1$ and $\mathcal{E}\left(\tilde{\alpha}_{f}, \tilde{\beta}_{f}\right)=1$ yielding

$$
\mathcal{C}\left(0, \tilde{\alpha}_{f} ; 0, \tilde{\beta}_{f}\right)=4,
$$

where we have associated as above $A= \pm 1$ to $H$ and $V$ polarization components and $B= \pm 1$ to positive and negative parity components. We will now see how these correlations

TABLE I. Probability table for a "nonlocal box" with two settings in the polarization ( $\alpha$ and $\left.\tilde{\alpha_{f}}\right)$ and 2 settings in the parity variable $(\beta$ and $\tilde{\beta_{f}}$ ).

\begin{tabular}{lllll}
\hline \hline $\begin{array}{l}\operatorname{pol}(\alpha) / \\
\operatorname{par}(\beta)\end{array}$ & \multicolumn{2}{c}{$\alpha=0$} & \multicolumn{2}{c}{$\alpha=\tilde{\alpha}_{f}$} \\
\hline$\beta=0$ & $I_{H \pi+}=0$ & $I_{H \pi-}=1 / 2$ & $I_{H \pi+}=1 / 2$ & $I_{H \pi-}=0$ \\
& $I_{V \pi+}=1 / 2$ & $I_{V \pi-}=0$ & $I_{V \pi+}=0$ & $I_{V \pi-}=1 / 2$ \\
$\beta=\tilde{\beta}_{f}$ & $I_{H \pi+}=1 / 2$ & $I_{H \pi-}=0$ & $I_{H \pi+}=1 / 2$ & $I_{H \pi-}=0$ \\
& $I_{V \pi+}=0$ & $I_{V \pi-}=1 / 2$ & $I_{V \pi+}=0$ & $I_{V \pi-}=1 / 2$ \\
\hline \hline
\end{tabular}

can be realized in principle with coupled degrees of freedom of classical light.

Let us start by writing the general form of matrices that only change the polarization or the parity angles. These are parametrized as follows:

$$
\mathcal{M}_{R}(\delta)=\left(\begin{array}{cccc}
a(\delta) & 0 & b(\delta) & 0 \\
0 & c(\delta) & 0 & d(\delta) \\
e(\delta) & 0 & f(\delta) & 0 \\
0 & g(\delta) & 0 & h(\delta)
\end{array}\right)
$$

and

$$
\mathcal{M}_{P}(\gamma)=\left(\begin{array}{cccc}
A(\gamma) & B(\gamma) & 0 & 0 \\
C(\gamma) & D(\gamma) & 0 & 0 \\
0 & 0 & E(\gamma) & F(\gamma) \\
0 & 0 & G(\gamma) & H(\gamma)
\end{array}\right)
$$

respectively. Here the functions $a(\delta), A(\gamma)$, etc., are at this point completely arbitrary complex functions of the angular parameters $\delta$ and $\gamma$. Let us assume that these functions are real. Then it is straightforward to impose the conditions in Table I for the relative intensities obtained from

$$
\mathcal{M}_{P}(\gamma) \mathcal{M}_{R}(\delta) v_{i}
$$

where $\delta$ takes the values $\alpha=0, \tilde{\alpha}_{f}, \gamma$ takes the values $\beta=$ $0, \tilde{\beta}_{f}$, while $v_{i}$ is taken the same as previously given by (15). One possible and convenient choice is

$$
\mathcal{M}_{R}(\alpha)=\left(\begin{array}{cccc}
\cos (\alpha) & 0 & -\sin (\alpha) & 0 \\
0 & \cos (\alpha) & 0 & -\sin (\alpha) \\
\sin (\alpha) & 0 & \cos (\alpha) & 0 \\
0 & \sin (\alpha) & 0 & \cos (\alpha)
\end{array}\right)
$$

and

$$
\mathcal{M}_{P}(\gamma)=\left(\begin{array}{cccc}
\cos 2 \gamma & \sin \gamma & 0 & 0 \\
\sin 2 \gamma & \cos \gamma & 0 & 0 \\
0 & 0 & \cos \gamma & \sin 2 \gamma \\
0 & 0 & \sin \gamma & \cos 2 \gamma
\end{array}\right)
$$

with $\tilde{\alpha}_{f}=\alpha_{f}=\pi / 2$ and $\gamma=\tilde{\beta}_{f}=\pi / 2$, while the identity operator corresponds to $\alpha=0$ and $\gamma=\beta=0$.

The matrix $\mathcal{M}_{R}(\alpha)$ is the same matrix seen above [Eq. (13)] that transforms (rotates) the polarization degree of freedom independently of the parity state [23]. It is thus factorizable and unitary:

$$
\begin{aligned}
\mathcal{M}_{R}\left(\tilde{\alpha}_{f}=\pi / 2\right) & =\left(\begin{array}{rrrr}
0 & 0 & -1 & 0 \\
0 & 0 & 0 & -1 \\
1 & 0 & 0 & 0 \\
0 & 1 & 0 & 0
\end{array}\right) \\
& =\left(\begin{array}{rr}
0 & -1 \\
1 & 0
\end{array}\right)^{\mathrm{pol}} \otimes\left(\begin{array}{ll}
1 & 0 \\
0 & 1
\end{array}\right)^{\mathrm{par}} .
\end{aligned}
$$

The parity transformation however is not unitary, as can be checked from the explicit matrix

$$
\mathcal{M}_{P}\left(\tilde{\beta}_{f}=\pi / 2\right)=\left(\begin{array}{rrrr}
-1 & 1 & 0 & 0 \\
0 & 0 & 0 & 0 \\
0 & 0 & 0 & 0 \\
0 & 0 & 1 & -1
\end{array}\right) .
$$


We can constrain nonunitarity to the parity degree of freedom by writing Eq. (39) as

$$
\begin{aligned}
\mathcal{M}_{P}\left(\tilde{\beta}_{f}=\pi / 2\right)= & \left(\begin{array}{rrrr}
1 & 0 & 0 & 0 \\
0 & 1 & 0 & 0 \\
0 & 0 & 0 & -1 \\
0 & 0 & -1 & 0
\end{array}\right) \\
& \times\left(\begin{array}{rrrrr}
-1 & 1 & 0 & 0 \\
0 & 0 & 0 & 0 \\
0 & 0 & -1 & 1 \\
0 & 0 & 0 & 0
\end{array}\right) .
\end{aligned}
$$

Indeed, the first matrix in Eq. (40) is unitary and given the initial states we are considering, this matrix leaves the polarization invariant but swaps the parity (with a $\pi$ phase shift) conditioned on the fact that the polarization is vertical. On the other hand the last matrix in Eq. (40) is obviously nonunitary and only acts on the parity degree of freedom [compare with Eq. (14)]; in parity space this matrix can be written as

$$
\left(\begin{array}{rr}
-1 & 1 \\
0 & 0
\end{array}\right)^{\mathrm{par}}
$$

Nonunitarity is a well-known key ingredient for violating Tsirelson's bound.

\section{B. Characterization of the extremal nonunitary gate in terms of quantum circuits}

Deterministic nonunitary gates are strictly forbidden by the quantum theory, because deterministic evolution obeys Schrödinger's equation which is unitary. It is however possible to realize a probabilistic nonunitary gate by coupling a quantum system to an ancilla. Such a protocol was suggested by Terashima and Ueda [17], who proposed a general method for implementing nonunitary gates in the context of quantum circuits theory. The main idea is to perform a generalized measurement [24] during which the system under interest is coupled with an ancilla and gets entangled with the latter. Finally, a local von Neumann (projective) measurement is performed onto the ancilla. Viewed at the level of the system, the whole procedure constitutes a generalized measurement or in technical terms, a POVM (positive operator valued measure). Due to entanglement between the system and the ancilla, the evolution undergone by the state of the system during the POVM is not unitary. It is also probabilistic in the sense that it depends on the particular result of the measurement undergone by the ancilla. This explains how Terashima and Ueda derived an optimal POVM aimed at probabilistically realizing an arbitrary nonunitary gate. In summary they showed the following results.

Let us consider a nonunitary gate operation defined as

$$
|\psi\rangle \rightarrow \frac{N|\psi\rangle}{\sqrt{\left\langle\psi\left|N^{\dagger} N\right| \psi\right\rangle}}
$$

with $N$ a nonunitary linear transformation, which is normalized through the condition

$$
\max _{|\psi\rangle}\left(\left\langle\psi\left|N^{\dagger} N\right| \psi\right\rangle\right)=1
$$

To implement (41), a generalized measurement [24] described by a set of operators $\left\{M_{m}\right\}$ is realized, during which, starting with the initial condition $|\psi\rangle\left|0^{\text {anc }}\right\rangle$, the state $\sum_{m}\left(M_{m}|\psi\rangle\right)\left|m^{\text {anc }}\right\rangle$ is reached. Thereafter, the ancilla undergoes a projective von Neumann measurement in the canonical ancilla basis $\left|m^{\text {anc }}\right\rangle$ yielding the postmeasurement state given

by $\frac{M_{m}|\psi\rangle}{\sqrt{\left\langle\psi\left|M_{m}^{\dagger} M_{m}\right| \psi\right\rangle}}$ with probability $p(m)=\left\langle\psi\left|M_{m}^{\dagger} M_{m}\right| \psi\right\rangle$.
The constraint

$$
\sum_{m} M_{m}^{\dagger} M_{m}=1
$$

ensures that the state $\sum_{m}\left(M_{m}|\psi\rangle\right)\left|m^{\text {anc }}\right\rangle$ is a physically reachable state. Incidentally, when this constraint is fulfilled, we find that

$$
\left(\left\langle\psi_{m}^{\dagger} M_{m} \mid \psi\right\rangle\right) \leqslant 1
$$

which ensures, together with (43), that $p(m)$ is a well defined probability distribution.

Note that the constraint

$$
\max _{|\psi\rangle}\left(\left\langle\psi_{m}^{\dagger} M_{m} \mid \psi\right\rangle\right) \leqslant 1
$$

is de facto verified, which explains a posteriori the significance of the condition (42). The probabilistic realization of the nonunitary transformation (41) requires us to perform a generalized measurement $\left\{M_{0}, M_{1}\right\}$ with two outcomes, 0 and 1 , such that $M_{0}=c N, M_{1}=\sqrt{I-M_{0}^{+} M_{0}}$ (in the sense $M_{1}^{\dagger} M_{1}=I-M_{0}^{+} M_{0}$ ), where $c$ is a normalization constant which follows, in accordance with (42) and (45),

$$
|c| \leqslant 1 \text {. }
$$

The successful measurement corresponds to outcome 0 , in which case the state is transformed according to

$$
|\psi\rangle \rightarrow \frac{M_{0}|\psi\rangle}{\sqrt{\left\langle\psi_{0}^{\dagger} M_{0} \mid \psi\right\rangle}}=\frac{\frac{c}{|c|} N|\psi\rangle}{\sqrt{\left\langle\psi^{\dagger} N \mid \psi\right\rangle}},
$$

as desired.

Otherwise, when outcome 1 is delivered, the measurement is unsuccessful. The probability to successfully implement the nonunitary transformation (41) is thus equal to

$$
p(|\psi\rangle, c)=\left\langle\psi_{0}^{\dagger} M_{0} \mid \psi\right\rangle=|c|^{2}\left\langle\psi^{\dagger} N \mid \psi\right\rangle .
$$

This probability is less than or equal to $|c|^{2}$. While the value of $c$ does not affect the postmeasurement state (which is pure because when the process is successful no summation over $m$ is performed), it affects the probability of success. The optimal measurement is reached when $|c|^{2}=1$.

Let us now reconsider the maximal violation of Tsirelson's bound. As we have seen above, Eq. (40) can be realized provided we are able to implement the nonunitary transformation

$$
\left(\begin{array}{rr}
-1 & 1 \\
0 & 0
\end{array}\right)^{\mathrm{par}}
$$


Making use of Eq. (42), we see that it corresponds to the properly normalized nonunitary gate

$$
\begin{aligned}
N^{\mathrm{par}} & =\frac{\left(\begin{array}{cc}
-1 & 1 \\
0 & 0
\end{array}\right)^{\mathrm{par}}}{\sqrt{\max _{|\psi\rangle}\left[\left\langle\psi\left|\left(\begin{array}{cc}
-1 & 0 \\
1 & 0
\end{array}\right)^{\mathrm{par}}\left(\begin{array}{cc}
-1 & 1 \\
0 & 0
\end{array}\right)^{\mathrm{par}}\right| \psi\right\rangle\right]}} \\
& =\frac{\left(\begin{array}{rr}
-1 & 1 \\
0 & 0
\end{array}\right)^{\mathrm{par}}}{\sqrt{\max _{|\psi\rangle}\left[\left\langle\psi\left|\left(\begin{array}{rr}
1 & -1 \\
-1 & 1
\end{array}\right)^{\mathrm{par}}\right| \psi\right\rangle\right]}}
\end{aligned}
$$

Since

$$
\left(\begin{array}{rr}
1 & -1 \\
-1 & 1
\end{array}\right)^{\text {par }}
$$

is twice the projector onto

$$
\frac{1}{\sqrt{2}}\left(\begin{array}{r}
1 \\
-1
\end{array}\right)^{\mathrm{par}},
$$

we obtain

$$
N=\frac{1}{\sqrt{2}}\left(\begin{array}{rr}
-1 & 1 \\
0 & 0
\end{array}\right)^{\mathrm{par}}
$$

If we choose $c=-1$, which is optimal because $|c|=1$, we find that

$$
\begin{aligned}
M_{0} & =\frac{1}{\sqrt{2}}\left(\begin{array}{rr}
1 & -1 \\
0 & 0
\end{array}\right)^{\mathrm{par}} ; M_{1}^{+} M_{1}=I-M_{0}^{+} M_{0} \\
& =\frac{1}{2}\left(\begin{array}{ll}
1 & 1 \\
1 & 1
\end{array}\right)^{\mathrm{par}} .
\end{aligned}
$$

Several choices for $M_{1}$ are possible, but setting

$$
M_{1}=\frac{1}{\sqrt{2}}\left(\begin{array}{ll}
0 & 0 \\
1 & 1
\end{array}\right)^{\text {par }}
$$

is particularly elegant because the corresponding generalised measurement can be realized by the equivalent of an analyzer in the "dual" basis

$$
\left\{\frac{1}{\sqrt{2}}\left(\begin{array}{c}
1 \\
-1
\end{array}\right)^{\mathrm{par}}, \quad \frac{1}{\sqrt{2}}\left(\begin{array}{l}
1 \\
1
\end{array}\right)^{\mathrm{par}}\right\},
$$

which is known in the context of quantum information to correspond to a Hadamard qubit gate. Actually the canonical basis

$$
\left\{\left(\begin{array}{l}
1 \\
0
\end{array}\right)^{\mathrm{par}},\left(\begin{array}{l}
0 \\
1
\end{array}\right)^{\mathrm{par}}\right\}
$$

and its dual basis are mutually unbiased [25] and maximally complementary in the qubit Hilbert space.

\section{Realization of the optimal nonunitary gate with Maxwell fields}

In principle, the analyzer that separates states belonging to a basis dual to the eigenbasis of the parity operator could be realized by a technique very similar to the one already implemented by the authors of Ref. [10] for analyzing parity;

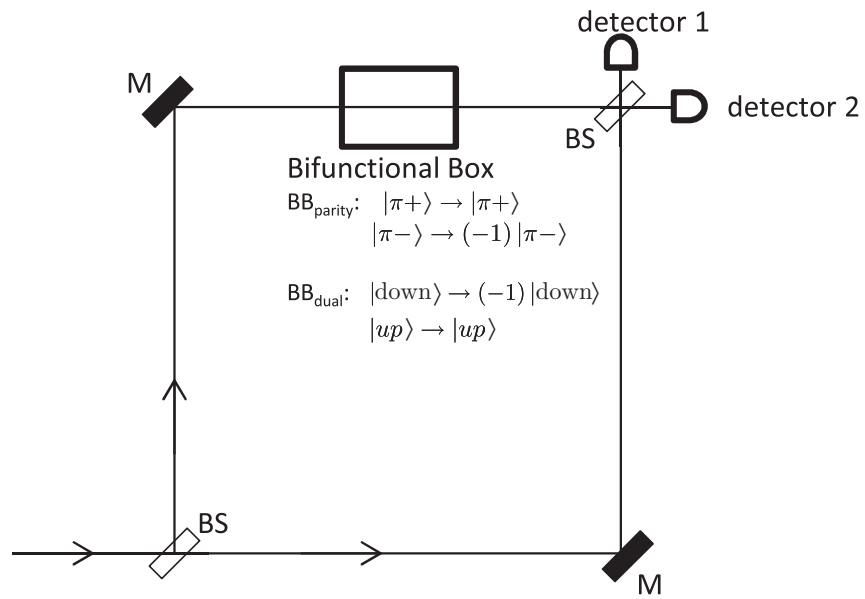

FIG. 1. Parity analyzer and its dual counterpart based on MachZehnder interferometry.

recall that the parity eigenbasis $\{|\pi+\rangle,|\pi-\rangle\}$ corresponds to the vectors

$$
\left\{\left(\begin{array}{l}
1 \\
0
\end{array}\right)^{\mathrm{par}},\left(\begin{array}{l}
0 \\
1
\end{array}\right)^{\mathrm{par}}\right\} .
$$

Indeed, eigenstates of the parity operator $|\pi+\rangle$ and $|\pi-\rangle$ can be separated in different outgoing channels by sending (see Fig. 1) the incoming beam into a Mach-Zehnder setup in which in one internal arm a phase shift $\pi$ is imposed to the states of odd parity $|\pi-\rangle$, while even-parity states $(|\pi+\rangle)$ remain unaffected. This operation corresponds, in matrix notation, to the Pauli $\sigma_{Z}$ matrix,

$$
\sigma_{Z}=\left(\begin{array}{rr}
1 & 0 \\
0 & -1
\end{array}\right)^{\mathrm{par}} .
$$

By doing so, one of the channels outside the interferometer (at the level of the detectors shown in Fig. 1) corresponds to the projector onto even-parity states and the other one to the projector onto odd-parity states.

Besides this, we must realize a similar device but this time in the dual basis, that we shall from now on denote the up-down basis for reasons that will become clear in the next section. The up-down basis is defined by

$$
\begin{aligned}
\mid \text { up }\rangle & =(|\pi+\rangle+|\pi-\rangle) / \sqrt{2}, \text { and } \mid \text { down }\rangle \\
& =7(|\pi+\rangle-|\pi-\rangle) / \sqrt{2} .
\end{aligned}
$$

It is easy to check that the same method just described for analyzing parity will work, but instead we must impose in one arm of the Mach-Zehnder a $\pi$ phase shift to the states |down $\rangle$ while the $\mid$ up $\rangle$ states remain unaffected (see Fig. 1). This operation corresponds to the Pauli $\sigma_{X}$ matrix:

$$
\begin{aligned}
\sigma_{X}= & \left(\begin{array}{ll}
0 & 1 \\
1 & 0
\end{array}\right)^{\mathrm{par}}=\left(\frac{1}{\sqrt{2}}\right)\left(\begin{array}{rr}
1 & 1 \\
1 & -1
\end{array}\right)^{\mathrm{par}} \\
& \times\left(\begin{array}{rr}
1 & 0 \\
0 & -1
\end{array}\right)^{\mathrm{par}}\left(\frac{1}{\sqrt{2}}\right)\left(\begin{array}{rr}
1 & 1 \\
1 & -1
\end{array}\right)^{\mathrm{par}} .
\end{aligned}
$$

In other words, we must permute the even and odd-parity eigenstates in one arm of the interferometer, which can be 
(a)

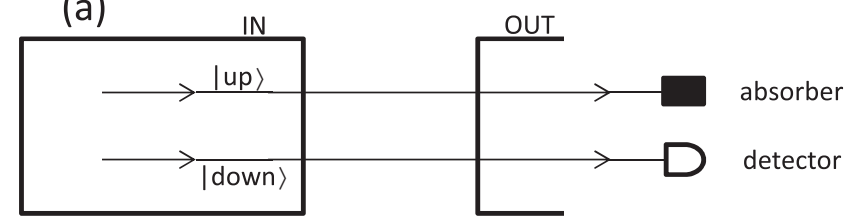

(b)

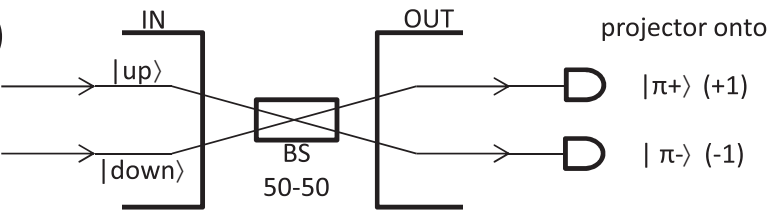

FIG. 2. (a) Realization of Eq. (49) with a nonunitary gate and (b) parity analyzer [Eq. (55)] in the double beam configuration.

done thanks to the $\operatorname{sgn}(x)$ transformation that we introduced above (Sec. III A).

In conclusion, depending on our choice to realize either (55) or (57) there correspond two different implementations of the experimental setup. In Figs. 1, 3, and 5, we represent the two options by a bifunctional box (BB) that can be realized in two different ways. Either nothing is done at the level of the bifunctional box, which corresponds to (55), or we perform a basis change thanks to a half wave plate, turning (55) into (57).

A simpler manner to implement these operations experimentally (as described immediately below in Sec. V) is to associate the (\{|up $\rangle, \mid$ down $\rangle\})$ basis to two spatially distinct beams of light, produced by the same laser source and propagating in the same directions (see Figs. 2 and 3) [26]. Then the up and down components can be separated (analyzed) by placing one detector along one beam and the other detector along the second beam. In order to realize the optimal POVM aimed at implementing the nonunitary transformation given by Eq. (40) the beam corresponding to the operator $M_{1}$ (which is actually the "outgoing channel" corresponding to the $\mid$ up $\rangle$ state) is discarded and one measures solely the intensity associated to the other beam (corresponding to the |down $\rangle$ state and operator $M_{0}$ ). In order to measure in the dual parity basis $(\{|\pi+\rangle,|\pi-\rangle\})$, we can in principle send the beams into the ingoing arms of a 50-50 beam splitter (see Fig. 2). We now describe the detailed experimental implementation of these schemes.

\section{EXPERIMENTAL (MAXIMAL) VIOLATION OF TSIRELSON'S BOUND}

\section{A. Beam preparation for maximum entanglement of polarization and parity degrees of freedom}

The optical setup used to prepare a Bell state is described in Fig. 3. We will see that the use of a linearly polarized laser beam $(\mathrm{He}-\mathrm{Ne})$ and of some basic optical elements is sufficient to prepare a maximally entangled state of the electromagnetic field. The beam from the laser is spatially cleaned and expanded with a spatial filter associated with a beam expander (SF/BE). This large (centimetric) and uniform beam with a high spatial coherence passes through two identical circular diaphragms ( $2 \mathrm{H}$ in Fig. 3) with a diameter of $2 \mathrm{~mm}$, disposed symmetrically relative to the main propagation axis $z$ at a distance $2 x_{0}$ of $8 \mathrm{~mm}$. Any component of the electromagnetic field can then be described by a distribution in the transverse plane composed of two functions of the variables $x$ and $y$ each being centered at $x_{0}(>0)$ and $-x_{0}$ (see Fig. 3). Thus, neglecting diffraction, the two beams can be well described by a transverse electromagnetic field which reads

$$
\vec{E}(x, y, z, t)=e^{i(k z-\omega t)}\left(\begin{array}{c}
E_{x}(x, y) \\
E_{y}(x, y) \\
0
\end{array}\right) .
$$

Thereafter the propagation term $e^{i(k z-\omega t)}$ will be omitted. Thus, at the first step of the setup (i.e., after the two holes and before the two half wave plates, Fig. 3) the field $\vec{E}$ can be analyzed with the Jones formalism (Sec. III A): $\vec{E}(x, y)$ is then expressed through $f_{\pi=1}(x, y) E$ where

$$
E=\left(\begin{array}{l}
a \\
b
\end{array}\right)
$$

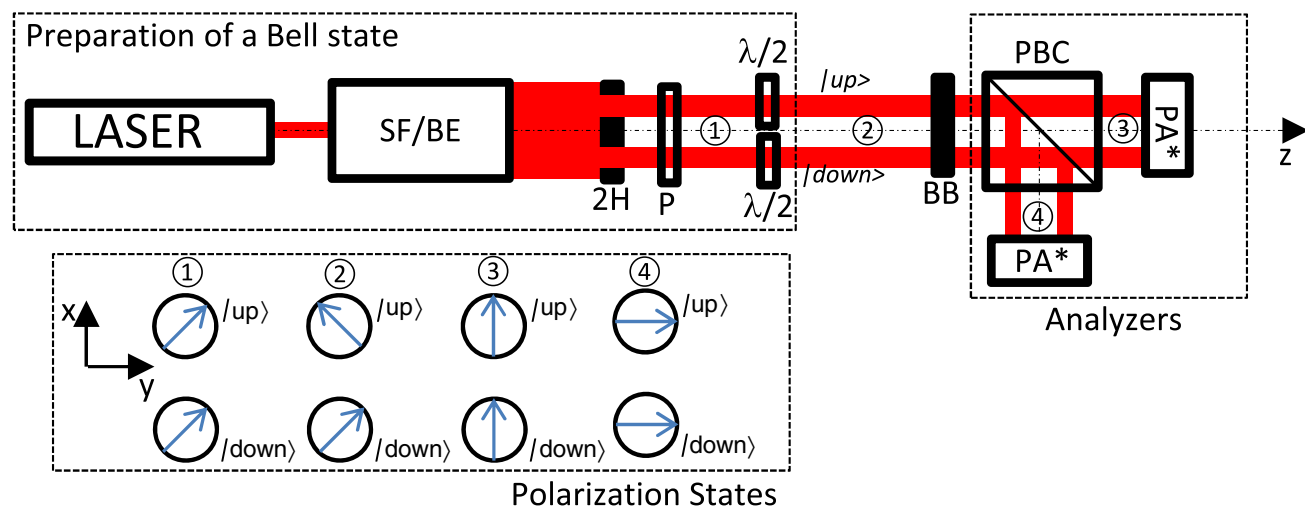

FIG. 3. Optical setup for preparing a Bell state which can be modified by a tunable device denoted bifunctional box (BB) and analyzed (i) in polarization with a polarizing beam splitter cube (PBC) and (ii) in parity (PA*) with a parity analyzer PA2 or a nonunitary gate PA1 (see Fig. 4). $P$ is a linear polarizer. SF/BE is a spatial filter used to spatially clean and expand the laser beam. $2 H$ is an aluminium plate perforated by two holes symmetric relative to the optical axis. $\frac{\lambda}{2}$ is a half wave plate. The inset "Polarization States" shows the beams (|up $\rangle$ and $\mid$ down $\rangle$ ) at each stage. 
is the Jones vector (normalized to unity) of the field $\vec{E}$ and $f_{\pi=1}(x, y)$ is an even function with respect to $x$. For convenience, we impose to the field $\vec{E}$ the normalization condition $\iint_{\infty}^{\infty}\left|f_{\pi=1}(x, y)\right|^{2} d x d y=1$. Phase plates locally acting on the polarization of the field are described by a Jones (unitary) matrix:

$$
M(\phi, \alpha)=\left(\begin{array}{cc}
\cos \left(\frac{\phi}{2}\right)+i \sin \left(\frac{\phi}{2}\right) \cos (2 \alpha) & i \sin \left(\frac{\phi}{2}\right) \sin (2 \alpha) \\
i \sin \left(\frac{\phi}{2}\right) \sin (2 \alpha) & \cos \left(\frac{\phi}{2}\right)-i \sin \left(\frac{\phi}{2}\right) \cos (2 \alpha)
\end{array}\right)
$$

where $\phi$ is the phase shift introduced by the birefringent plate between the two orthogonal eigenvectors and $\alpha$ is the angle of rotation of these eigenaxes relatively to the axes of the laboratory $(x$ and $y$ ) (as an example, a half wave plate is characterized by a phase shift $\phi=\pi$, while $\alpha$ is fixed by our choice of the orientation of the plate).

\section{B. Maximally entangled beam}

In our experiment the initial state obeys

$$
E_{0}=\left(\begin{array}{l}
a_{1} \\
b_{1}
\end{array}\right)=\left(\begin{array}{l}
\frac{1}{\sqrt{2}} \\
\frac{1}{\sqrt{2}}
\end{array}\right)
$$

It is simple to show that from $E_{0}$, one can construct a maximally entangled state, by using two half wave plates $(\lambda / 2)$ (see Fig. 3). A first half wave plate characterized by an angle $\alpha_{1}=0$ acts on the upper beam whereas the second one characterized by an angle $\alpha_{2}=\frac{\pi}{4}$ acts on the bottom beam, as expressed in the following equation:

$$
E_{i}=\left(\begin{array}{l}
a_{2} \\
b_{2}
\end{array}\right)=N\left(\alpha_{1}, \alpha_{2}\right)\left(\begin{array}{l}
a_{1} \\
b_{1}
\end{array}\right)
$$

with the generalized Jones matrix

$$
\begin{aligned}
N\left(\alpha_{1}, \alpha_{2}\right)= & \frac{1}{2}[1+\operatorname{sgn}(x)] M\left(\pi, \alpha_{1}\right) \\
& +\frac{1}{2}[1-\operatorname{sgn}(x)] M\left(\pi, \alpha_{2}\right) .
\end{aligned}
$$

This yields the entangled state

$$
E_{i}=\frac{1}{\sqrt{2}}\left(\begin{array}{c}
1 \\
-\operatorname{sgn}(x)
\end{array}\right)
$$

i.e., the maximally entangled state considered in Sec. III A [Eq. (11)].

\section{Classical analysis of parity and parity sensing}

In the configuration of the setup described in Fig. 3, the two beams exiting the element denoted $2 H$ are completely disjoint; the amplitude characterizing the incident field $\vec{E}$ can be consequently written as $f_{\pi=1}(x, y)=(1 / \sqrt{2}) g\left(x-x_{0}, y\right)+$ $(1 / \sqrt{2}) g\left(x+x_{0}, y\right)$ where $g(x, y)$ is the spatial distribution of amplitude of each beam respectively centered at $x_{0}$ and $-x_{0}$. Note that since $g\left(x-x_{0}, y\right)$ and $g\left(x+x_{0}, y\right)$ are defined on two disjoint supports, we have $g\left(x-x_{0}, y\right) g\left(x+x_{0}, y\right)=0$. Superpositions of parity eigenstates are described by a function $G(x, y)=a_{+} f_{\pi=1}(x, y)+a_{-} \operatorname{sgn}(x) f_{\pi=1}(x, y)$, which can be rewritten as follows:

$$
\begin{aligned}
G(x, y)= & \left(a_{+}+a_{-}\right)(1 / \sqrt{2}) g\left(x-x_{0}, y\right) \\
& +\left(a_{+}-a_{-}\right)(1 / \sqrt{2}) g\left(x+x_{0}, y\right)
\end{aligned}
$$

with $\left|a_{+}\right|^{2}+\left|a_{-}\right|^{2}=1$. We then define two coefficients characterizing the distribution between the up and down beams through $A_{\text {up }}=\frac{1}{\sqrt{2}}\left(a_{+}+a_{-}\right)$and $A_{\text {down }}=\frac{1}{\sqrt{2}}\left(a_{+}-a_{-}\right)$. The link between the parity and up-down amplitudes is indeed given via the Hadamard transformation, as required by Eq. (56),

$$
\left(\begin{array}{l}
a_{+} \\
a_{-}
\end{array}\right)=\frac{1}{\sqrt{2}}\left(\begin{array}{cc}
1 & 1 \\
1 & -1
\end{array}\right)\left(\begin{array}{c}
A_{\text {up }} \\
A_{\text {down }}
\end{array}\right) .
$$

\section{Analysis of entangled states}

The polarization analyzer used in our setup is a polarization beam splitter cube (PBC in Fig. 3) that transmits the horizontal polarization and reflects the vertical one. Figure 4 shows the nonunitary gate (PA1) and the parity analyzer (PA2) implemented in our setup. Whereas PA1 is exactly the nonunitary gate

$$
\left(\begin{array}{rr}
1 & -1 \\
0 & 0
\end{array}\right)
$$

described in Fig. 2 of the preceding Sec., PA2 is slightly different from the unitary gate denoted $\sigma_{Z}$ in the parity eigenbasis given by Eq. (55). Our parity analyzer (PA2) is instead based on Young's two holes interference. The accuracy of our measurements is limited by the ability to locate the position of the interference fringes on the camera: a dark (respectively bright) fringe located in the center of our camera corresponds to an odd (even) state ( $\pi-$ and $\pi+$ respectively). The principle of our parity analyzer PA2 will be scrutinized in the next paragraph.

\section{E. Interferometric parity analyzer (PA2)}

To study the parity of an unknown entangled state we have to measure the two complex coefficients $a_{+}$and $a_{-}$described in Sec. VC. In our setup a lens is used to focus the two beams on a charge-coupled device (CCD) sensor of a camera in order to obtain an intensity image of the resulting interference. This image is proportional to the norm squared of the Fourier transform of the incident electric field (before the lens). In our setup, this electric field is completely described by the function

PA 1

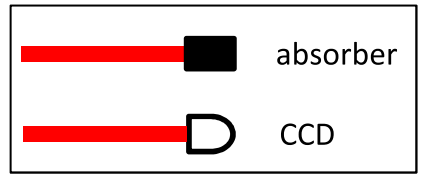

FIG. 4. Nonunitary gate (PA1) and parity analyzer (PA2).
PA 2

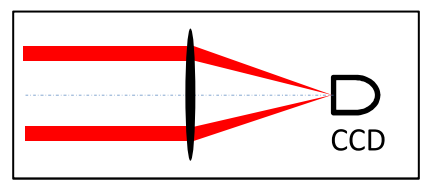


empty BB

half wave plate

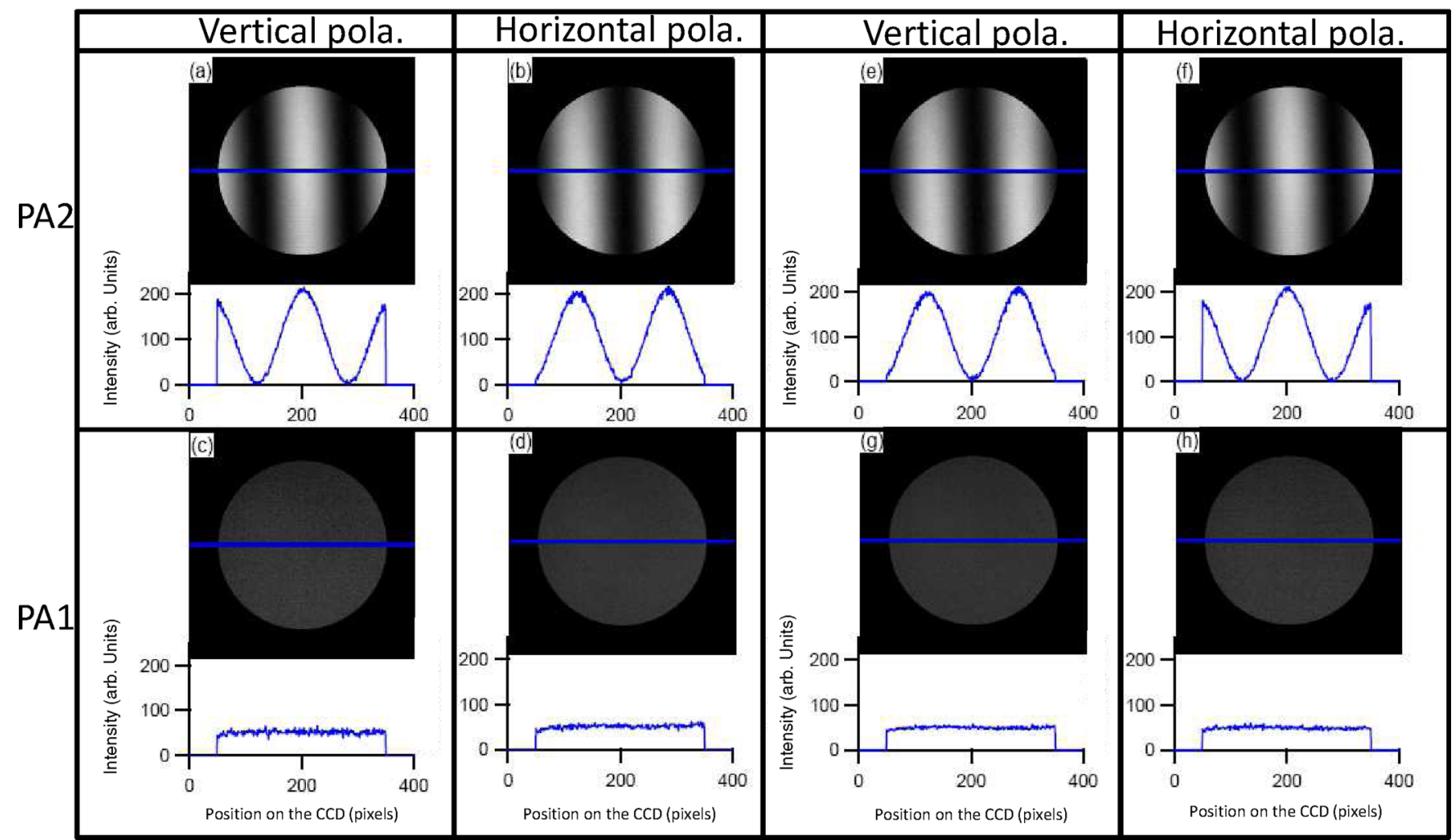

FIG. 5. (a)-(d) Experiments realized with an empty bifunctional box (BB). (e)-(h) Experiments realized with the bifunctional box (BB) being a half wave plate in order to rotate polarizations by an angle of $\frac{\pi}{2}$. (a),(e) and (b),(f) are parity measurements (PA2 in Fig. 4) for respectively the vertical and horizontal polarizations (3 and 4 in Fig. 3). (c),(g) and (d),(h) are nonunitary measurements (PA1 in Fig. 4) for respectively the vertical and horizontal polarizations (3 and 4 in Fig. 3).

$G(x, y)[$ Eq. (65)], and the intensity image is thus proportional to $\left|\hat{G}\left(\kappa_{x}, \kappa_{y}\right)\right|^{2}$ with

$$
\hat{G}\left(\kappa_{x}, \kappa_{y}\right)=\iint_{\infty}^{\infty} G(x, y) e^{-2 i \pi\left(\kappa_{x} x+\kappa_{y} y\right)} d x d y,
$$

where $\kappa_{x}$ and $\kappa_{y}$ are the spatial frequencies defined by $\kappa_{x}=\frac{x^{\prime}}{\lambda f}$ and $\kappa_{y}=\frac{y^{\prime}}{\lambda f}$ with $f$ the focal length of the lens, $\lambda$ is the wavelength of the source, and $\left(x^{\prime}, y^{\prime}\right)$ are the coordinates in the Fourier plane (recorded in the focal plane of the lens). We then obtain

$$
\begin{aligned}
\left|\hat{G}\left(\kappa_{x}, \kappa_{y}\right)\right|^{2}= & \left|\hat{g}\left(\kappa_{x}, \kappa_{y}\right)\right|^{2}\left[\left|A_{\text {up }}\right|^{2}+\left|A_{\text {down }}\right|^{2}+2\left|A_{\text {up }}\right|\left|A_{\text {down }}\right|\right. \\
& \left.\times \cos \left(2 \pi \kappa_{x} x_{0}-\Delta \psi\right)\right]
\end{aligned}
$$

where $\Delta \psi=\arg \left(A_{\text {up }} A_{\text {down }} *\right)$ is the phase shift between the complex amplitudes $A_{\text {up }}$ and $A_{\text {down }}$. Note that we have chosen all parameters of our setup to have the function $\left|\hat{g}\left(\kappa_{x}, \kappa_{y}\right)\right|$ quasiconstant around the center of the camera. Thus we can easily extract from our images (see Fig. 5) two independent measures: the visibility

$$
V=\frac{|\hat{G}|_{\max }^{2}-|\hat{G}|_{\min }^{2}}{|\hat{G}|_{\max }^{2}+|\hat{G}|_{\min }^{2}} \approx \frac{2\left|A_{\mathrm{down}}\right|\left|A_{\mathrm{up}}\right|}{\left|A_{\mathrm{down}}\right|^{2}+\left|A_{\mathrm{up}}\right|^{2}}
$$

of the fringes and the relative intensity

$$
I_{0}=\frac{2|\hat{G}(0,0)|^{2}}{|\hat{G}|_{\max }^{2}+|\hat{G}|_{\min }^{2}}=1+V \cos (\Delta \psi)
$$

at the center of the camera $\left(x^{\prime}=0\right)$. The ratio $\left|A_{\text {up }}\right| /\left|A_{\text {down }}\right|$ is deduced from $V$ and $\Delta \psi$ from $I_{0}$; finally using Eq. (66) we obtain the two coefficients $a_{+}$and $a_{-}$and are thus able to estimate the parity intensities $\left|a_{+}\right|^{2}$ and $\left|a_{-}\right|^{2}$.

\section{F. Results}

In our experiment we have realized two kinds of measuresusing the nonunitary gate (PA1) for the first one and the parity analyzer (PA2) for the second one as described in Fig. 4-for different implementations of the bifunctional box (BB) (see Fig. 3). In Fig. 5, images (a)-(d) have been obtained with an empty bifunctional box (BB) whereas images (e)-(h) have been recorded with a half wave plate as $\mathrm{BB}$ in order to rotate the polarization of the two beams by an angle of $\frac{\pi}{2}$. The results of the nonunitary measures deliver an average intensity corresponding to probabilities equal to $0.4980,0.5019,0.4996$, and 0.5003 respectively for images (c), (d), (g), and (h). We estimated visually the measurement uncertainties on each individual pixel to be close to 0.03. As the intensities are estimated after averaging over a large number of pixels, we finally obtain probabilities very close to 0.5 . The parity analysis provides two pieces of information: the visibility of 
TABLE II. Intensities associated to the four experimental settings leading to maximal violation of Cirelson bound.

\begin{tabular}{lcccc}
\hline \hline $\operatorname{pol}(\alpha) / \operatorname{par}(\beta)$ & \multicolumn{2}{c}{$\alpha=0($ empty BB) } & $\alpha=\tilde{\alpha}_{f}$ (BB: half wave plate) \\
\hline$\beta=0$ (PA2) & $I_{H \pi+}=0.007$ & $I_{H \pi-}=0.493$ & $I_{H \pi+}=0.493$ & $I_{H \pi-}=0.007$ \\
& $I_{V \pi+}=0.493$ & $I_{V \pi-}=0.007$ & $I_{V \pi+}=0.007$ & $I_{V \pi-}=0.493$ \\
$\beta=\tilde{\beta}_{f}$ (PA1) & $I_{H \pi+}=0.5019$ & $I_{H \pi-}=0$ & $I_{H \pi+}=0.5003$ & $I_{H \pi-}=0$ \\
& $I_{V \pi+}=0$ & $I_{V \pi-}=0.4980$ & $I_{V \pi+}=0$ & $I_{V \pi-}=0.4996$ \\
\hline \hline
\end{tabular}

the fringes $V$ which is equal to 0.972 with an uncertainty close to 0.03 in all cases (a), (b), (e), and (f) and the relative intensity at the center of the camera $I_{0}$ equal to 1.97 for images (a) and (f) and 0.03 for images (b)and (e) with an uncertainty close to 0.03 too. Thus we can calculate the amplitude ratio $\frac{\left|A_{\text {up }}\right|}{\left|A_{\text {down }}\right|}=\frac{1 \pm \sqrt{1-V^{2}}}{V}=0.784$ in all cases and the phase shift $\Delta \psi$ equal to 0 for (a) and (f) and $\pi$ for (b) and (e). Using Eq. (66) we obtain the parity vectors

$$
\left(\begin{array}{l}
a_{+} \\
a_{-}
\end{array}\right)=\left(\begin{array}{c}
0.993 \\
-0.120
\end{array}\right)
$$

for (a) and (f) and

$$
\left(\begin{array}{c}
0.120 \\
-0.993
\end{array}\right)
$$

for (b) and (e).

Finally, we obtain the experimental table, Table II, to be compared with its theoretical counterpart given by Table I. It is worth noting that the four values 0 which appear in the line of Table II corresponding to $\beta=\tilde{\beta}_{f}$ do represent the null intensity "measured" at the output of the absorber in Fig. 2, which in turn corresponds to the second line of the matrix

$$
\left(\begin{array}{cc}
-1 & 1 \\
0 & 0
\end{array}\right)^{\mathrm{par}}
$$

of Eq. (40). The non-null values were obtained by averaging the intensities collected with a CCD camera. The corresponding value of the $\mathrm{CHSH}$ function is then equal to

$$
0.986+0.986+0.9999+0.9999=3.97
$$

with an error of less than $3 \%$.

\section{DISCUSSION}

\section{A. Nature of correlations}

The first point that should be clear is that violating, in the present context, a Bell-type inequality is unrelated to nonlocality [2] (in particular it would be misleading to infer, as has sometimes been done in the past [27], that Maxwell fields exhibit some type of nonlocal features). The reason, as is well known, is that Bell's theorem is a mathematical statement that does not rely on any quantum-mechanical ingredient [28], nor for that matter on locality. The theorem is grounded on a factorization assumption for hidden variable probability distributions, which in the specific EPR setup was taken by Bell to be a consequence of local causality [19].

Here instead the violation of a Bell-type inequality rules out the existence of a so-called noncontextual model for light according to which (i) the state of the electromagnetic field would be described as a statistical distribution of elementary components ("little balls");

(ii) each little ball would be characterized by a hidden variable $\lambda$ that determines its behavior when polarization and parity operations are performed, e.g., whether after a rotation in polarization (or parity) spaces, the "little ball" will end up in the $H / V$ (or $\pi+/ \pi-$ ) branches of the beam.

The factorization assumption means then that for a "little ball" described by $\lambda$, the polarization and parity properties are independent, i.e., its final polarization state only depends on $\lambda$ and on the polarization operations, while its final parity state only depends on $\lambda$ and on the parity operations [29]. The factorization assumption implies that the correlation function $\mathcal{C}\left(\alpha, \alpha^{\prime} ; \beta, \beta^{\prime}\right)[$ Eq. (26)] depending on the average intensities is bounded by 2 .

The fact that this Bell inequality can be violated implies that for our little balls, the factorization assumption does not hold and that its polarization properties also depend on the parity operations. While a hidden-variable description for Maxwell fields is less compelling than for quantum mechanics (essentially because classical fields do not lead to discrete measurement outcomes), the tools employed to quantify the correlations have nevertheless turned out to be useful to investigate the statistical features of classical light fields [8-13]. The focus is then shifted to which type of states of the electromagnetic field gives rise to violations of Bell inequalities, and to which point the coupled degrees of freedom are correlated. The violations can be interpreted as follows: even though they are local fields, Maxwell fields do share with the quantum-mechanical state a holistic feature, namely that the different degrees of freedom are nonseparable properties, just as operations performed on a single particle in an entangled multiparticle state affect the entire wave function.

\section{B. Nonunitarity and postselection}

In this work, we have presented results showing a violation of Tsirelson's bound based on the implementation of a nonunitary gate. In a quantum context, it is well known that nonunitary evolution induces a statistical bias [30]. Indeed, nonunitarity is related to losses, and if the statistics are renormalized by discarding the losses, an apparent violation may occur; for instance it may possible not only to violate Tsirelson's bound with quantum entangled systems [30], but also to violate a Bell-CHSH inequality with quantum systems prepared in a separable state [32] and/or local systems, as well as with statistical distributions of classical systems [31].

Nonunitary quantum systems have recently $[30,33,34]$ been employed in order to simulate "superquantum" correlations and investigate their properties. Typically this is done by 
exploiting polarization-dependent losses in photonic setups employing entangled pairs of photons. The correlations are simulated by renormalizing the real ("naked") probabilities so as to compensate for losses at the level of the detectors. In Ref. [34], the maximal violation that was reached was 3.42 (as compared with the maximal bound of 4) and optimality, in the sense of $|c|=1$ in Eq. (46), was not attained in that case. In Ref. [33] the region of extremal violation characteristic of PR boxes was indeed reached, and also in that limit a key ingredient was that one of the channels was totally lossy [30], as in our implementation. Seemingly, this is a necessary ingredient for reaching extremal violation of the Tsirelson bound.

We are here in a similar situation but with classical fields: if instead of measuring PA1 - the nonunitary gate

$$
\left(\begin{array}{rr}
1 & -1 \\
0 & 0
\end{array}\right)
$$

described in Fig. 2-we would have measured the outcomes of the unitary gate

$$
\left(\begin{array}{rr}
1 & -1 \\
1 & 1
\end{array}\right),
$$

implying no losses, Tsirelson's bound would not have been violated.

Our results thus indicate that Maxwell fields may also be an interesting tool in order to simulate different types of correlations, and they may even offer more flexibility than quantum systems. Actually, if we attenuated the laser used in our experiment in order to reach the quantum regime (viewed here as the "few photons at a time" regime), we would also obtain an extremal violation of Tsirelson's bound, as we now explain.

\section{Linking the quantum and classical regimes}

While it has been remarked recently that the common features found in classical fields and quantum mechanics are afforded by the Hilbert spaces employed in both cases [4], it is tempting to attribute this quantum type of behavior of Maxwell fields to the ultimate quantum field nature of light. As we shall show elsewhere, the Glauber correlation function $[35,36]$ is in the present context a very useful tool for establishing a link between the classical, Maxwell regimes (many photons) and the quantum regime (single photon).

The key ingredient can be found in a paper by Glauber and Titulaer [36], who considered among others situations during which the quantum state of light is a coherent state, something that occurs whenever the source is a laser source, as was the case in our experiments. Roughly, they established the following property: when light is prepared in a coherent state, the amplitude of probability to detect a first photon at time $t_{1}$ and position $\mathbf{r}_{1}$, a second one at time $t_{2}$ and position $\mathbf{r}_{2}, \ldots$ an $i$ th photon at time $t_{i}$ and position $\mathbf{r}_{i} \ldots$ and a $N$ th photon at time $t_{N}$ and position $\mathbf{r}_{N}$ factorizes into the product of $N$ equivalent functions $\mathbf{E}\left(\mathbf{r}_{i}, t_{i}\right)$. We wrote here these functions in bold because they are three-component functions, reflecting the fact that the detectors are sensitive in principle to the direction of polarization of the incoming light. Besides, it can be shown that this function $\mathbf{E}(\mathbf{r}, t)$ behaves as a classical electric field in the following sense [37,38]: it propagates in vacuum and more generally in all media with a linear response as a classical electric field, it transforms under the Lorentz group as the classical electric field, and, in good approximation, it couples to matter as the electric field would do.

This indicates that the renormalized intensities appearing in our derivation of a maximal violation of Tsirelson's bound also possess a quantum flavor: if we put a coherent absorber at the output of the laser in order to reach the singlephoton regime, and perform measurements with single-photon detectors instead of continuous ones, we can expect that the statistics will be, up to noise, exactly the same as in the classical regime. Considered so, the aforementioned connection between contextuality and violation of Bell's inequalities (mentioned in Sec. VI A) is also valid in the quantum regime. Hence even in the strongly attenuated, single-photon regime, our setup makes it possible to reveal the intrinsic contextuality of light, to be understood in this regime in a purely quantum context.

\section{CONCLUSIONS}

In summary, we have employed entangled states of classical Maxwell fields coupling the polarization and spatial parity degrees of freedom in order to implement the analog of nonunitary transformations, leading to maximal correlations beyond Tsirelson's bound. A nonunitarity gate was obtained by implementing an optimal POVM [17]. Moreover, as has been discussed in Sec. VIC, by virtue of the Glauber-Titulaer factorization property, we expect that our device would also lead to a maximal violation of $\mathrm{CHSH}$ inequalities in the (quantum) single-photon regime, in line with recent quantum optics experiments [33,34].

From this point of view, the use of the aforementioned dictionary between entangled quantum and classical systems was doubly useful. The characterization of nonunitarity in terms of generalized measurements [17,24] allowed us to conceive an optimal setup for maximally violating the $\mathrm{CHSH}$ inequality in the classical regime (Maxwell fields, many photons). Translated to the single-photon regime, these results could provide a technique for optimally producing "supercontextual" correlations. An advantage of using Maxwell fields is that one precisely knows the physical properties of the beam that is manipulated.

It is not clear whether there exist practical applications of a system that exhibits PR correlations, in quantum and classical optics as well. All that we can say at this level is that a violation of Tsirelson bound (with classical and/or quantum light as well) is the mark of nonunitarity.

\section{ACKNOWLEDGMENTS}

The authors acknowledge support from the "Mission pour l'interdisciplinarité" C.N.R.S., France (PEPS project APPLIQUANT: L' optique appliquée: un champ d' expérimentation pour les théories quantiques et super-quantiques). One of us (T.D.) acknowledges fruitful and enriching discussions with an unrecognized precursor of the violation of Bell's inequalities by classical fields, the late signore De Nigris. 
[1] E. Schroedinger, Proc. Cambridge Philos. Soc. 31, 555 (1935).

[2] R. J. C. Spreeuw, Phys. Rev. A 63, 062302 (2001).

[3] P. Ghose and M. K. Samal, arXiv:quant-ph/0111119.

[4] P. Ghose and A. Mukherjee, Rev. Theor. Sci. 2, 274 (2014).

[5] A. F. Abouraddy, T. Yarnall, B. E. A. Saleh, and M. C. Teich, Phys. Rev. A 75, 052114 (2007).

[6] M. A. Goldin, D. Francisco, and S. Ledesma, J. Opt. Soc. Am. B 27, 779 (2010).

[7] C. V. S. Borges, M. Hor-Meyll, J. A. O. Huguenin, and A. Z. Khoury, Phys. Rev. A 82, 033833 (2010).

[8] P. Chowdhury, A. S. Majumdar, and G. S. Agarwal, Phys. Rev. A 88, 013830 (2013).

[9] X.-F. Qian and J. H. Eberly, Opt. Lett. 36, 4110 (2011).

[10] K. H. Kagalwala, G. Di Giuseppe, A. F. Abouraddy, and B. E. A. Saleh, Nat. Photon. 7, 72 (2013).

[11] L. J. Pereira, A. Z. Khoury, and K. Dechoum, Phys. Rev. A 90, 053842 (2014)

[12] M. McLaren, T. Konrad, and A. Forbes, Phys. Rev. A 92, 023833 (2015).

[13] X. F. Qian, B. Little, J. C. Howell, and J. H. Eberly, Optica 2, 611 (2015).

[14] B. S. Cirelson, Lett. Math. Phys. 4, 93 (1980).

[15] L. J. Landau, Phys. Lett. A120, 54 (1987).

[16] S. Popescu and D. Rohrlich, Found. Phys. 24, 379 (1995).

[17] H. Terashima and M. Ueda, Int. J. Quantum Inf. 03, 633 (2005).

[18] J. F. Clauser, M. A. Horne, A. Shimony, and R. A. Holt, Phys. Rev. Lett. 23, 880 (1969); J. F. Clauser and A. Shimony, Rep. Prog. Phys. 41, 1881 (1978).

[19] J. S. Bell, in In Between Science and Technology, edited by A. Sarlemijn and P. Kroes (Elsevier, Amsterdam, 1990), reprinted in J. S. Bell, Speakable and Unspeakable in Quantum Mechanics, 2nd Ed. (Cambridge University Press, Cambridge, England, 2004).

[20] N. Gisin, Phys. Lett. A154, 201 (1991).

[21] In principle, the action of a Jones matrix is the same everywhere in the plane transverse to the propagation direction of light. Here two Jones matrices are simultaneously implemented: one in the positive $x$ region and the other in the negative $x$ region.

[22] It is not appropriate to talk about nonlocality in the case of Maxwell fields as we discussed in Sec. VI, but we stick however to the commonly accepted denomination, as explained above.
[23] The choices (36) and (37) are not unique; other forms for these matrices can be considered if they are better suited to an experimental realization $[30,33]$.

[24] E. B. Davies and J. T. Lewis, Commun. Math. Phys. 17, 239 (1970).

[25] T. Durt, B.-G. Englert, I. Bengtsson, and K. Zyczkowski, Int. J. Quantum Inf. 08, 535 (2010).

[26] It is straightforward to associate to this preparation process a qubit state $\alpha|u p\rangle+\beta \mid$ down $\rangle$. This can be done without any ambiguity in the case of quasimonochromatic beams, having in mind that $|\alpha|^{2} /|\beta|^{2}$ is the ratio of the ingoing intensities and the dephasing between the two beams is the dephasing between $\alpha$ and $\beta$.

[27] G. De Nigris, Nuovo Cimento B 108, 17 (1993).

[28] A. Matzkin, in Foundations of Probability and Physics 5, edited by L. Accardi, G. Adenier, C. Fuchs, G. Jaeger, A. Yu. Khrennikov, J.-A. Larsson, and S. Stenholm, AIP Conf. Proc. No. 1101 (AIP, Melville, NY, 2009), p. 339.

[29] If the hidden variables are deterministic, the final polarization or parity state depends on $\lambda$, while for stochastic hidden variables, the probability for reaching a given final state depends on $\lambda$.

[30] D. W. Berry, H. Jeong, M. Stobinska, and T. C. Ralph, Phys. Rev. A 81, 012109 (2010).

[31] A. Matzkin, J. Phys. A 41, 085303 (2008).

[32] This is known in the literature as the detection loophole, which has important consequences for what concerns the experimental confirmation of nonlocality [39]. It has also implications in quantum cryptography where nonlocality is a tool for thwarting Trojan horse attacks [40].

[33] M. Ringbauer, A. Fedrizzi, D. W. Berry, and A. G. White, Sci. Rep. 4, 6955 (2014).

[34] D. S. Tasca, S. P. Walborn, F. Toscano, and P. H. Souto Ribeiro, Phys. Rev. A 80, 030101 (2009).

[35] R. J. Glauber, Phys. Rev. 130, 2529 (1963).

[36] U. M. Titulaer and R. J. Glauber, Phys. Rev. 140, B676 (1965).

[37] J. E. Sipe, Phys. Rev. A 52, 1875 (1995).

[38] B. J. Smith and M. G. Raymer, New J. Phys. 9, 414 (2007).

[39] J. A. Larsson, J. Phys. A 47, 424003 (2014).

[40] T. Durt, Phys. Rev. Lett. 86, 1392 (2001); 83, 2476 (1999). 